\title{
Evaluación de los niveles sonoros generados por el parque automotor en el centro urbano, Chachapoyas, Perú
}

\section{Evaluation of the sound levels generated by the automotive park in the urban center Chachapoyas, Peru}

\author{
Atilio Ramos Chappa ${ }^{1}$
}

\section{RESUMEN}

El estudio tuvo como objetivo evaluar los niveles sonoros dentro del centro urbano de la ciudad, donde se identificó los puntos de monitoreo en las intersecciones de las calles. Se analizó los niveles de sonido encontrados y se elaboró un mapa de contaminación sonora, en el cual se identificaron 27 puntos y se midió el sonido con un sonómetro tipo II. Los resultados obtenidos fueron comparados con los Estándares de Calidad Ambiental (ECA) establecidos por el Ministerio del Ambiente. Se encontró que los puntos ubicados dentro de la zona de protección especial superan los parámetros, opuesto a los puntos de la zona comercial que no lo superan, sin embargo, en la zona residencial solo tres puntos $\left(\mathbf{P}_{2}, \mathbf{P}_{6} \mathbf{y} \mathbf{P}_{21}\right)$ de 13 no superan los ECA, también se observó que el máximo nivel de presión sonora se encontró durante el medio día en el punto $\mathbf{P}_{4}=\mathbf{6 9 . 4} \mathrm{dB}$. Estos resultados se plasmaron en un mapa de ruido para el centro urbano de la ciudad. Se concluyen que los altos niveles sonoros están determinados por el flujo vehicular, tubo de escape liberado y vehículos pesados.

Palabras clave: Niveles sonoros, contaminación sonora

\begin{abstract}
The objective of the study was to evaluate sound levels within the urban center of the city, where monitoring points were identified at street intersections. The sound levels found were analyzed and a noise pollution map was drawn up, in which 27 points were identified and the sound was measured with a type II sound level meter. The results obtained were compared with the Environmental Quality Standards (ECA) established by the Ministry of the Environment. It was found that the points located within the special protection zone exceed the parameters, opposite to the points of the commercial zone that do not exceed it, however, in the residential area only three points (P2, P6 and P21) of 13 do not exceed the RCTs, it was also observed that the maximum sound pressure level was found during the middle of the day at the point $\mathrm{P} 4=69.4 \mathrm{~dB}$. These results were reflected in a noise map for the urban center of the city. It is concluded that high noise levels are determined by vehicular flow, released exhaust pipe and heavy vehicles.
\end{abstract}

Keywords: Sound levels, noise pollution 


\section{INTRODUCCIÓN}

El contaminante ruido, es un tema de gran importancia para el mundo, debido a que afecta la salud de todos los seres vivos que se encuentran expuestos a este contaminante de forma continua, la contaminación sonora, es generado principalmente por las construcciones, industrias, comercio, animales domésticos, el parque automotor y espectáculos sociales, estos dos últimos también llamados ruido comunitario o ambiental (Ram \& Dom, 2011).

El ruido ambiental, es la más fácil de generar pero la más difícil de controlar (Bello Martinez, 2009), en las grandes ciudades la principal fuente de contaminación sonora viene siendo emitida por el parque automotor que engloba a todos los vehículos motorizados que circulan por las calles (Alfie y Salinas, 2016), esto se evidencia por el crecimiento demográfico y el uso desmedido de los vehículos.

La contaminación sonora ocurre en un determinado tiempo y espacio definido y su manifestación termina cuando se silencia la fuente de emisión (Ram \& Dom, 2011), estar expuesto constantemente al ruido conlleva a la pérdida de audición, cuyas personas más vulnerables son los mayores de 45 años (Ballesteros et al., 2012). La deficiencia auditiva inducida por el ruido viene siendo un mal común que aqueja a un gran porcentaje de personas a nivel mundial, debido a que no provoca efectos inmediatos visibles ni se otorga las consideraciones de un riesgo laboral, esto explica parcialmente la mayor pérdida auditiva en personas de mayor edad o tiempo de exposición que estos llevan en un trabajo (Ballesteros et al., 2012).

El flujo vehicular, la proporción de vehículos pesados, la ausencia de silenciador en el tubo de escape, motor viejo o antiguo y la velocidad de desplazamiento; son variables que inciden en el aumento del nivel de sonido, ante esto se puede afirmar que a mayor número de vehículos que emiten el mismo nivel de sonido el valor final se incrementa (Calvo, Álvarez, San Román y Cobo, 2012); esto se puede evidenciar con el estudio que realizo Ram \& Dom, (2011), donde muestra que dos vehículos que emiten el mismo nivel de sonido, incrementan el valor final en $3 \mathrm{dBA}$; en relación a la velocidad de desplazamiento, si se dobla la velocidad, el nivel de ruido se incrementara de 9 a $12 \mathrm{dBA}$.

En los últimos años la ciudad de Chachapoyas, Perú, ha presentado un incremento acelerado del parque automotor, superando los mil vehículos entre particulares y los que hacen servicio de taxi, y a este se suman los otros tipos de vehículos que circulan por la ciudad (MPCH, 2017).

Es por ello que se desarrolló este estudio en el centro urbano de la ciudad de Chachapoyas, conocido también como ambiente urbano, con el objetivo de evaluar los niveles sonoros que genera el parque automotor en los diferentes espacios públicos.

\section{MATERIAL Y MÉTODOS}

El diseño es descriptivo de una sola casilla. Para la identificación de los puntos a evaluar se optó por la metodología de viales o de tráfico (Ministerio del Ambiente Perú, 2013). La muestra estuvo conformada por 27 puntos pertenecientes a fuentes móviles. Se realizó un muestreo no probabilístico. El estudio se desarrolló en dos fases.

El trabajo de campo consistió en recabar toda la información necesaria, como la identificación de los puntos a monitorear de acuerdo al flujo vehicular y los espacios públicos especiales, todo ello en un horario punta, la georreferenciación de cada punto de monitoreo se realizó con un GPS (Sistema de Posicionamiento Global), se monitoreo los niveles sonoros en cada punto con un sonómetro tipo II durante 10 minutos en los tres horarios establecidos y los siete días de la semana, simultáneamente se realizó el conteo de vehículos pesados, livianos y motocicletas lineales que circularon en cada punto de monitoreo.

En el trabajo de gabinete, se procedió a evaluar los datos obtenidos en campo de cada punto para determinar si existe o no contaminación sonora por cada punto monitoreado, para ello se procedió a analizar los datos, donde se calculó la media aritmética para cada día y por toda la semana con la siguiente formula:

$$
\begin{array}{ll}
X=\frac{\sum_{i=1}^{n} X i}{n} & \begin{array}{l}
\text { Donde: } \\
\mathrm{X}: \text { Media Aritmética. } \\
\mathrm{X}_{\mathrm{i}}: \text { i-ésimo valor observado. } \\
\mathrm{n}: \text { es el número de muestras }
\end{array}
\end{array}
$$

Con la ayuda del D.S. N.085-2003-PCM donde expresa los valores de los Estándares de Calidad Ambiental ECA para las diferentes zonas económicas de una ciudad según determina la municipalidad, se procedió a comparar los resultados de la media aritmética de toda la semana de nuestro estudio, el cual se analizó cuidadosamente para determinar si existe o no contaminación sonora en cada punto evaluado previo análisis, los resultados fueron plasmados en un mapa de contaminación sonora para la ciudad en tres horarios diurno (mañana, medio día y tarde), además de un mapa general de toda la semana en el cual se identificaron los puntos o áreas que alcanzan el máximo nivel sonoro. 


\section{RESULTADOS}

Tabla 1

Promedio de niveles sonoros en función al tráfico vehicular por semana medidos en 10 minutos

\begin{tabular}{|c|c|c|c|c|}
\hline \multirow[t]{2}{*}{$\begin{array}{l}N^{\circ} \text { de } \\
\text { punto }\end{array}$} & \multicolumn{3}{|c|}{$\begin{array}{c}\text { Promedio dxehículos } \\
\text { por semanan } 10 \\
\text { minutos. }\end{array}$} & \multirow[t]{2}{*}{ LA eq } \\
\hline & $\mathbf{V P}$ & VL & ML & \\
\hline \multicolumn{5}{|c|}{ Valores Mayores } \\
\hline $\mathbf{P 4}$ & 4.9 & 121.3 & 42.4 & 68.3 \\
\hline P8 & 4.1 & 130.3 & 41.8 & 67.2 \\
\hline P 17 & 2.4 & 121.7 & 26.4 & 66.2 \\
\hline \multicolumn{5}{|c|}{ Valores Menores } \\
\hline $\mathbf{P 2}$ & 2.0 & 28.1 & 10.2 & 59.4 \\
\hline P6 & 1.3 & 25.8 & 11.5 & 57.8 \\
\hline P10 & 0.2 & 9.0 & 2.1 & 54.2 \\
\hline
\end{tabular}

\section{Donde:}

VP: Vehículos Pesados.

VL: Vehículos Livianos.

ML: Motocicletas Lineales.

Laeq: valores del nivel sonoro en decibeles (dBA)

Los resultados muestran que los niveles sonoros están en función al tráfico vehicular, es por ello que se encontró mayor nivel sonoro en los puntos con mayor tráfico como el punto 04,08 y 17, sin embargo, los menores valores encontrados fueron en los puntos con menor tráfico vehicular, como es el punto 02,06 y 10. En el siguiente cuadro se muestra el número total de vehículos de cada tipo que pasan durante los 10 minutos y los valores de nivel sonoro por cada punto

\section{Figura 1}

Valores del nivel sonoro (LAeq) promedio en cada punto vs los estándares de calidad ambiental (ECA)

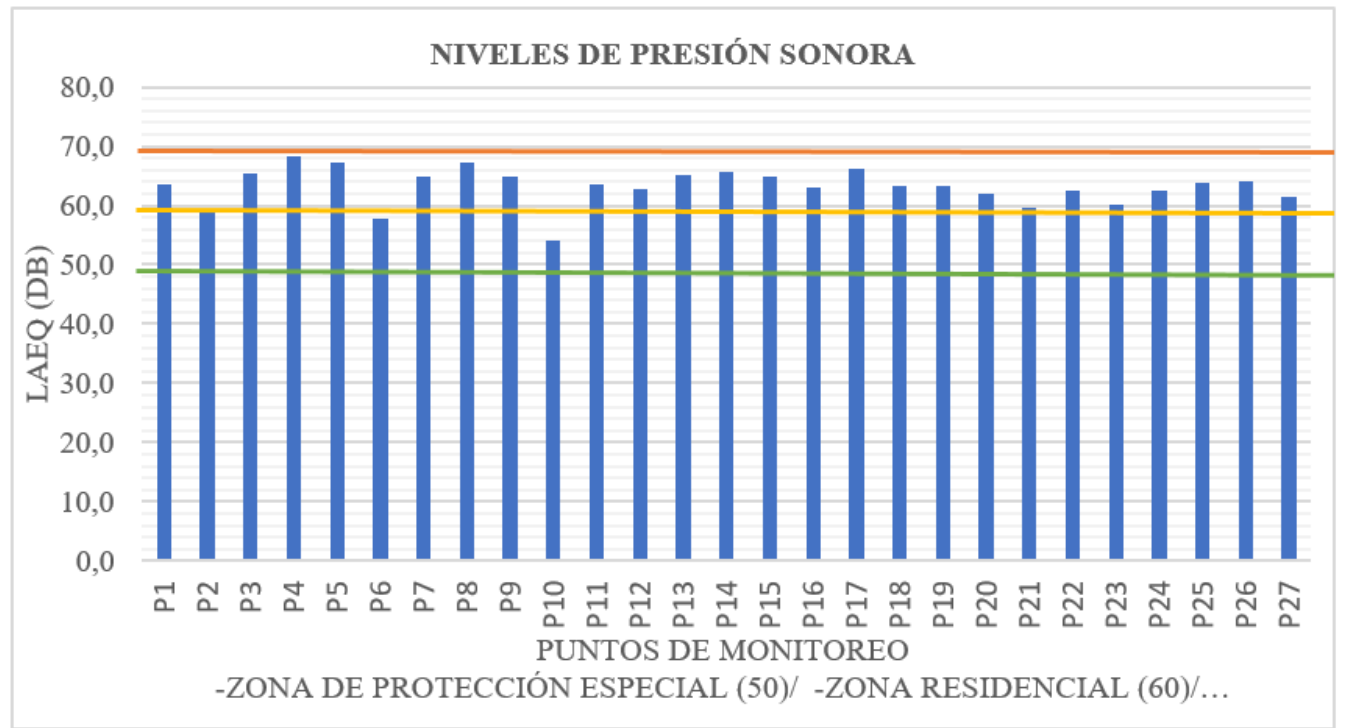

Luego de obtener la media aritmética de toda la semana por cada punto se procedió a comparar con los Estándares de Calidad Ambiental (ECA) diurno establecidos por el MINAM, donde se encontró que; los puntos $\mathbf{P}_{5}, \mathbf{P}_{7}, \mathbf{P}_{9}, \mathbf{P}_{13}$ $\mathbf{P}_{19}, \mathbf{P}_{22}, \mathbf{P}_{23}, \mathbf{P}_{24}, \mathbf{P}_{25} \mathbf{y} \mathbf{P}_{26}$ exceden el ECA Residencial, a excepción de los puntos $\mathrm{P}_{2}, \mathrm{P}_{6}$ y $\mathrm{P}_{21}$ que no superan los $60 \mathrm{~dB}$, los puntos $\mathbf{P}_{\mathbf{1}}, \mathbf{P}_{3}, \mathbf{P}_{4}, \mathbf{P}_{\mathbf{8}}, \mathbf{P}_{\mathbf{1 2}}, \mathbf{P}_{\mathbf{1 4},} \mathbf{P}_{\mathbf{1 5 y}} \mathbf{P}_{\mathbf{2 7}}$ no exceden el ECA Comercial y los puntos $\mathbf{P}_{\mathbf{1 0}} \mathbf{P}_{\mathbf{1 1}}, \mathbf{P}_{\mathbf{1 6}}, \mathbf{P}_{\mathbf{1 7}}, \mathbf{P}_{\mathbf{1 8 y}} \mathbf{P}_{\mathbf{2 0}}$ exceden el ECA de protección especial como se muestra en el siguiente cuadro. 


\section{Figura 2}

Mapa de ruido ambiental de toda la semana.
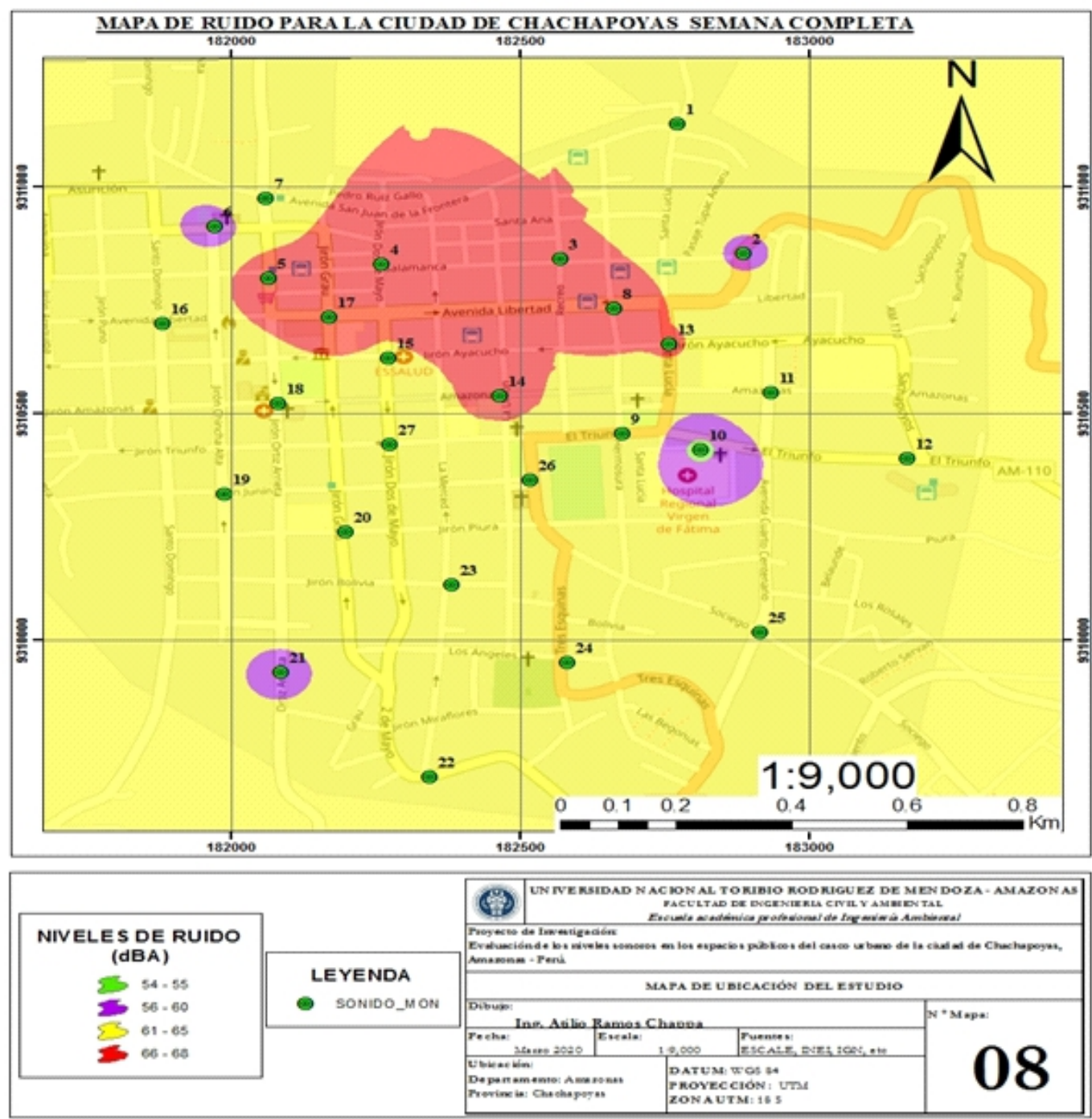

\section{DISCUSIÓN}

Según Mendoza, et al., (2018). mencionan en un estudio que la principal fuente de contaminación sonora en una ciudad proviene del parque automotor y los componentes de la contaminación está relacionado con el uso desmedido de la bocina, la falta de silenciador en los tubos de escape y los vehículos antiguos., entonces de acuerdo a este estudio se puede verificar que los vehículos que generan altos niveles sonoros son las motocicletas lineales con el tubo de escape liberado, vehículos viejos, camiones, volquetes y el uso excesivo del claxon, todo esto nos conlleva a coincidir con el autor.

Según el estudio realizado por Calvo, et al., (2012); el flujo vehicular y la velocidad de desplazamiento; son variables que inciden en el aumento del nivel de ruido, ante esto ellos afirman que a mayor número de vehículos que emiten el mismo nivel de sonido el valor final se incrementa, por ejemplo en dos vehículos el valor final aumenta en 3dBA., entonces de acuerdo al estudio realizado se coincide con este autor, ya que se encontró que los niveles sonoros son mayores en los puntos con mayor tráfico vehicular en relación a los puntos que alcanzaron los valores más bajos donde el tráfico vehicular es menor.

Para el análisis de las mediciones obtenidas, se realizó comparaciones con el D.S No. 085-2003PCM., donde se identifica que los puntos ubicados dentro de la zona especial supera los ECA en un $100 \%$, del mismo modo la zona residencial el $77 \%$ de los puntos evaluados superan los ECA.

De esta manera se coincide con Correa y Jara (2014) quienes determinaron que el sector Norte de la Ciudad de Loja presenta contaminación acústica sobrepasando los $65 \mathrm{~dB}$, de esto los autores afirman que el parque automotor es la fuente principal, 
sumado a esto la falta de conciencia ambiental de la ciudadanía y de los conductores.

Torres y Roncal (2015), determinaron el nivel de ruido por influencia del parque automotor en el cercado de Pucallpa. El resultado promedio obtenido fue de $77,61 \mathrm{~dB}$ el cual indica que supera en un $10 \%$ al ECA para una zona Comercial $(70 \mathrm{~dB})$, sin embargo, en este estudio se determinó que no existe contaminación sonora para la zona comercial, ya que los niveles sonoros encontrados no superan los ECA establecidos por el Ministerio del Ambiente (MINAM).

Según Carrillo (2012), la importancia de elaborar un mapa acústico radica en que ayuda a prevenir la exposición al ruido ambiental y a identificar áreas más adecuadas para una vida tranquila.

Maya et al.,(2010); indica que los mapas de ruido son muy importantes puesto que son utilizados como herramientas de gestión. Con este estudio se ha logrado coincidir con ambos autores, puesto que con la elaboración de un mapa de contaminación sonora utilizando el método de interpolación IDW (Inverse Distance Weighting), para la ciudad de Chachapoyas se identificó zonas más adecuadas para residir lejos de la contaminación sonora.

\section{CONCLUSIÓN}

Luego de evaluar las mediciones obtenidas en los puntos de monitoreo del centro urbano de la ciudad de Chachapoyas, se encontró que los puntos con mayor tráfico vehicular alcanzaron el mayor nivel sonoro opuesto a los puntos con menor tráfico, además los niveles sonoros responden a los componentes del parque automotor como es la antigüedad, el tamaño, tubo de escape liberado y el toque innecesario de la bocina.

\section{REFERENCIAS BIBLIOGRÁFICAS}

Alfie, M., y Salinas, O. (2016). Contaminación auditiva y ciudad caminable. Revista Técnico Ambiental.

Bello Martinez, W. (2009). Evaluación de los niveles de contaminación acústica del centro de la ciudad de Talca Evaluation of the noise levels in downtown Talca. Interamerican Journal of Environment and Tourism, 5(1), 1-10.

Calvo, J.A., Álvarez, C., San Román, J.L., y Cobo, P. (2012). Influence of vehicle driving parameters on the noise caused by passenger cars in urban traffic. Transportation Research Part D 17 (7), 509-513. Recuperado de http://www.scien cedirect.com/science/ article/pii/S136192091

2000582, doi 10.101 6/j. trd.2012.06.002.

Carrillo, J. D. (2012). A comparison of interpolation methods for creating noise maps in urban environments une comparaison des methodes d' interpolation pour la, 3(1), 62-68.

Correa, E.F., y Jara, M.F. (2014). Evaluación de la contaminación acústica derivada del parque automotor en el sector norte de la ciudad de Loja. (Proyecto de pregrado: Ingeniería en Manejo y Conservación del Medio Ambiente) Universidad Nacional de Loja, Ecuador. 133 p

Decreto supremo N ${ }^{\circ}$ 085-2003-PCM. Reglamento de estándares Nacionales de calidad ambiental para ruido. Recuperado de http://infoaire. minam.gob.pe:1013/INFOAIRE/archivos/nor mativa/RUIDO/DS _085-2003-PCM_Regla mento ECA para Ruido.pdf.

Maya, G., Correa, V. M., \& Gómez, O. M. (n.d.). Gestión para la prevención y mitigación del ruido urbano, (C).

Mendoza, É. C., Luis, J., Laurencio, L., Mauricio, R., \& Apaza, C. (2018). Determinación del nivel de presión sonora generada por el parque automotor en Ilo , Perú 1, 13(2), 14-20. https://doi.org/10.22507/pml.v13n2a2

Ministerio del Ambiente Perú. (2013). Resolucion Ministerial 227-2013-MINAM, (1013), 36. htt ps://doi.org/10.1017/CBO9781107415324.004

Ram, A., \& Dom, A. (n.d.). Medio ambiente, el ruido vehicular urbano : problemática agobiante de los países en vías de desarrollo, (42).

Torres, G.A., y Roncal, J.J. (2015). Evaluación de la calidad del aire por influencia del parque automotor en el cercado de Pucallpa distrito de Calleria, provincia de Coronel Portillo, departamento de Ucayali-2015. (Proyecto de pregrado: Ingeniería Ambiental). Universidad Nacional de Ucayali. 159 p.

Urbano, D. (2013). Plan de desarrollo urbano de la ciudad de Chachapoyas. 